\title{
Editorial: Developing and Deploying Negative Emission Technologies: System-Level Assessment and Rationalization
}

\author{
Miguel Brandão ${ }^{1}$, Patrick Lamers' ${ }^{2}$, Barbara Olfe-Kraeutlein ${ }^{3}$, Wilfried Rickels ${ }^{4}$, \\ Volker Sick ${ }^{5}$, Mijndert Van Der Spek ${ }^{6}$ and Aidong Yang ${ }^{7 *}$ \\ ${ }^{1}$ Department of Sustainable Development, Environmental Science and Engineering, Royal Institute of Technology, \\ Stockholm, Sweden, ${ }^{2}$ National Renewable Energy Laboratory, Golden, CO, United States, ${ }^{3}$ Group of CO2 Utilisation \\ Strategies and Society, Institute for Advanced Sustainability Studies, Potsdam, Germany, ${ }^{4}$ Faculty of Economics and Social \\ Sciences, Institute for World Economics, Christian Albrechts University Kiel, Kiel, Germany, ${ }^{5}$ Department of Mechanical \\ Engineering, University of Michigan, Ann Arbor, MI, United States, ${ }^{6}$ School of Engineering \& Physical Sciences, Heriot-Watt \\ University, Edinburgh, United Kingdom, ${ }^{7}$ Department of Engineering Science, University of Oxford, Oxford, United Kingdom
}

Keywords: carbon dioxide removal, negative emission technologies, system-level, lifecycle analysis, technoeconomic analysis, policy, deployment strategy, transition pathways

\section{Editorial on the Research Topic}

\section{OPEN ACCESS}

Edited and reviewed by: Phil Renforth,

Heriot-Watt University,

United Kingdom

*Correspondence: Aidong Yang

aidong.yang@eng.ox.ac.uk

Specialty section:

This article was submitted to Negative Emission Technologies,

a section of the journal

Frontiers in Climate

Received: 10 May 2021 Accepted: 01 June 2021

Published: 30 June 2021

Citation:

Brandão $M$, Lamers $P$ Olfe-Kraeutlein B, Rickels W, Sick $V$, Van Der Spek M and Yang A (2021) Editorial: Developing and Deploying

Negative Emission Technologies:

System-Level Assessment and Rationalization. Front. Clim. 3:707872.

doi: 10.3389/fclim.2021.707872
Developing and Deploying Negative Emission Technologies: System-Level Assessment and Rationalization

Climate change, induced by the excessive amount of greenhouse gas (GHG) emissions from anthropogenic activities, is one of the greatest global challenges of our times. To address this challenge, a range of important measures are being developed or have already been adopted, including switching from fossil fuels to renewable energy resources, reducing emissions through improving efficiencies and demand management, and capturing $\mathrm{CO}_{2}$ at point sources with subsequent storage to avoid their release to the atmosphere. In addition to measures curbing new emissions, intentional atmospheric carbon dioxide removal (CDR) by negative emissions technologies (NETs) is increasingly considered as necessary for compliance with ambitious temperature targets. According to the Intergovernmental Panel on Climate Change (2018), all climate pathways that limit global warming to $1.5^{\circ} \mathrm{C}$ with controlled (or no) overshoot project the use of CDR in the order of $100-1,000 \mathrm{GtCO}_{2}$ over the twenty first century. The deployment of NETs, removing $\mathrm{CO}_{2}$ from the atmosphere and storing it on land, underground, or in the oceans, could become one of the most significant undertakings in industrial development, with profound impacts on the future of our society.

NETs include a diverse range of options, such as bioenergy with carbon capture and storage (BECCS), direct air capture (DAC) and subsequent sequestration (DACCS) or use in products with long lifetimes, such a construction material, enhanced weathering and increasing ocean alkalinity, afforestation, and other land/soil management solutions (Royal Society and Royal Academy of Engineering, 2018; National Academy of Sciences Engineering Medicine, 2019). These options differ widely in their approach to capturing atmospheric carbon (such as biological vs. abiotic) and to storing the captured carbon (such as above ground, in soil, below the subsurface, or in the sea; and with or without chemical conversion). 
Complementary to the technical research and development of these schemes through experimental and pilot explorations, there is emerging research on important system aspects, such as the overall technoeconomic, environmental and social viability of individual NETs, policy requirements, potential synergies and conflicts with other climate actions (such as emission reduction), strategies for deploying NETs (where and when), and the integration of NETs and the associated industries with the wider economy.

Focusing on these systems issues, this Research Topic aimed to promote research and discussion on systematic approaches to the future deployment of NETs. At present, such approaches are particularly relevant for assessing their feasibility at the national level. Through examining the long-term low GHG emission development strategies from 16 countries plus one from the EU, Thoni et al. report the broad recognition of the role of NETs. However, their work show that existing feasibility assessments have mostly focused on technical and biophysical perspectives and lack social and cultural considerations. The authors highlight the need for further assessing pathways involving NETs to reflect upon challenges beyond climate mitigation, including socioeconomic goals. They argue that the outcome of more holistic feasibility assessments would be highly desirable to underpin the viability of NETs, especially when they are included in integrated assessment models (IAMs) and related trajectories for long-term climate mitigation and adaptation scenarios.

Focusing on UK land use and agriculture, Reay echoes the importance of carefully considering and taking actions along multiple perspectives, such as governance, finance, skills and society, in addition to research and development, in order to avoid pitfalls of NETs and other net zero initiatives caused by the lack of concerted and inclusive measures. Field also addresses bio-based systems and revisits the notion of "additional carbon," calling for increased attention to the difference between alternative carbon abatement systems with respect to carbon additionality, with more reliable accounting of ecosystematmosphere exchanges to complement existing approaches, such as LCA and supply-chain assessment.

Addressing the interplay between NETs and power generation, the work by Lehtveer and Emanuelsson on comparing BECCS and DACCS shows that, although DACCS has a higher levelized cost for carbon capture, its greater flexibility may lead to a lower total system (carbon removal + power generation) cost if NETs were to integrate with a power sector dominated by variable and intermittent renewables which would favor flexible demand. This study offers a concrete example illustrating the importance

\section{REFERENCES}

Intergovernmental Panel on Climate Change (2018). Global Warming of $1.5^{\circ} \mathrm{C}$. An IPCC Special Report on the Impacts of Global Warming of $1.5^{\circ} \mathrm{C}$ Above PreIndustrial Levels and Related Global Greenhouse Gas Emission Pathways, in the Context of Strengthening the Global Response to the Threat of Climate Change.

National Academy of Sciences Engineering and Medicine (2019). Negative Emissions Technologies and Reliable Sequestration. Washington, DC: National Academies Press. of integrative thinking between the deployment of NETs and other sectors.

Despite their potential importance, technology-readiness levels of some and total deployment levels of all NETs are still low, with a gradual deployment process yet to unfold along with other technological and socioeconomic transitions. Therefore, understanding the possible trajectories of co-evaluation with other sectors may provide important insights about the future of NETs. In this respect, the LCA study by Rosental et al. on carbon capture (including DAC) and utilization (CCU) shows that the reduction of negative environmental impacts of producing largevolume organic chemicals from captured carbon to a large extent depends on the progression in the sustainability of resource extraction, processing and recycling of materials (e.g., steel, aluminum and concrete) that form the technical infrastructure for CCU. In a separate discussion, Hastings and Smith argue that achieving net zero emissions would benefit significantly from the knowledge, skills, and assets of the oil and gas industry, and that seeking to play an active role in the deployment of NETs could in turn contribute to transforming the sector, which itself is facing an enormous challenge of transitioning to a sustainable future (Wilkinson et al., 2021).

In summary, the articles in this Research Topic are offering a valuable starting point for advocating and developing systematic approaches to the future progression of NETs. This is deemed to be part of a greater societal transition, where interactions between different perspectives and sectors will increasingly require robust system-level assessment and rationalization. We envisage that contributions will be particularly valued from future work focusing on the assessment of spatial and temporal deployment strategies, understanding of synergies and tradeoffs, development of tools to support inclusive decisions, and regional case studies that harness interdisciplinary strengths. Furthermore, research in these areas needs to be facilitated by the progress in LCA and technoeconomic assessment tools and standards to enable sound choices of boundary conditions and data, as these are essential for achieving rigorous, consistent and transparent results when assessing various NET or CDR schemes (Sick et al., 2020; Wilcox et al., 2021).

\section{AUTHOR CONTRIBUTIONS}

All authors listed have made a substantial, direct and intellectual contribution to the work, and approved it for publication. 
Conflict of Interest: The authors declare that the research was conducted in the absence of any commercial or financial relationships that could be construed as a potential conflict of interest.

The handling editor declared a shared affiliation with one of the authors, $\mathrm{MV}$, at time of review.
Copyright (c) 2021 Brandão, Lamers, Olfe-Kraeutlein, Rickels, Sick, Van Der Spek and Yang. This is an open-access article distributed under the terms of the Creative Commons Attribution License (CC BY). The use, distribution or reproduction in other forums is permitted, provided the original author(s) and the copyright owner(s) are credited and that the original publication in this journal is cited, in accordance with accepted academic practice. No use, distribution or reproduction is permitted which does not comply with these terms. 